\title{
Spatial specificity of tactile enhancement during reaching
}

\author{
Dimitris Voudouris $^{1}$ (D) $\cdot$ Katja Fiehler $^{1}$
}

Published online: 24 July 2017

(C) The Psychonomic Society, Inc. 2017

\begin{abstract}
Tactile signals on a hand that serves as movement goal are enhanced during movement planning and execution. Here, we examined how spatially specific tactile enhancement is when humans reach to their own static hand. Participants discriminated two brief and simultaneously presented tactile stimuli: a comparison stimulus on the left thumb or little finger from a reference stimulus on the sternum. Tactile stimuli were presented either during right-hand reaching towards the left thumb or little finger or while holding both hands still (baseline). Consistent with our previous findings, stimuli on the left hand were perceived stronger during movement than baseline. However, tactile enhancement was not stronger when the stimuli were presented on the digit that served as reach target, thus the perception across the whole hand was uniformly enhanced. In experiment 2 , we also presented stimuli on the upper arm in half of the trials to reduce the expectation of the stimulus location. Tactile stimuli on the target hand, but not on the upper arm, were generally enhanced, supporting the idea of a spatial gradient of tactile enhancement. Overall, our findings argue for low spatial specificity of tactile enhancement at movement-relevant body parts, here the target hand.
\end{abstract}

Keywords Tactile perception · Enhancement · Reaching · Discrimination thresholds

Dimitris Voudouris

dimitris.voudouris@psychol.uni-giessen.de

1 Experimental Psychology, Justus-Liebig University Giessen, Otto-Behaghel-Strasse 10F, 35394 Giessen, Germany

\section{Introduction}

To perform a goal-directed hand movement, we need constantly to know the location of our moving hand and the movement target. Typically, the location of our hand is determined by somatosensory and peripheral visual information. Indeed, when people perform goal-directed hand movements they shift their gaze to the target (Smeets, Hayhoe, \& Ballard, 1996; Johansson, Wrestling, Backstrom, \& Flanagan, 2001; Voudouris, Smeets, \& Brenner, 2016) instead of tracking their own hand movement. For this reason, the location of the movement target is typically derived through visual signals. Nevertheless, humans are also able to guide hand movements towards unseen targets by relying on somatosensory signals, for instance reaching towards an itchy spot at the back.

The strength of somatosensory modulation seems to be functionally specific. Somatosensory signals are attenuated when they arise on a limb that is about to move or is moving (Chapman, Bushnell, Miron, Duncan, \& Lund, 1987; Buckingham, Carey, Colino, deGrosbois, \& Binsted, 2010; Parkinson et al., 2011; Voss, Ingram, Wolpert, \& Haggard, 2008). This has been suggested to occur because humans cancel the re-afferences that can be predicted based on an internal forward model (Wolpert \& Flanagan, 2001; but see also Williams \& Chapman, 2002). Yet, tactile signals can also be enhanced (Eimer, Forster, van Velzen, \& Prabhu, 2005; van Ede, van Doren, Damhuis, de Lange, \& Maris, 2015). For example, tactile stimuli are detected faster when they arise at the target of an upcoming saccade (Rorden, Greene, Sasine, \& Baylis, 2002) or when they are presented on a finger that is about to move (Juravle \& Deubel, 2009). Tactile sensitivity can be increased on a limb that is moving to explore a surface manually (Juravle, McGlone, \& Spence, 2013), i.e., if somatosensory signals are relevant for the task. On a neural level, 
increased amplitudes of N140 ERP components in response to tactile stimulation have been found on a moving hand both before (Forster \& Eimer, 2007) and during reaching to the other hand (Juravle, Heed, Spence, \& Roder, 2016), which have been associated with tactile enhancement.

Recently, we showed that tactile signals are enhanced at body parts that are relevant for reaching movements (Voudouris \& Fiehler, 2017). In this previous study, participants performed right-hand reaches to the thumb of their left unseen hand that was still or to an external visual target. Participants had to detect a tactile stimulus that was presented either on the left little finger or on their right index finger. We found decreased tactile thresholds for stimuli presented on the left static hand but only when reaching to that hand and not when reaching to the external visual target. Importantly, tactile thresholds on the left hand when reaching to that hand were also decreased compared with a baseline, in which no reaching movement was performed. We suggested that the lower tactile thresholds resulted from an increase in perceived intensity of the tactile stimulus, supporting the notion of tactile enhancement.

In the study by Voudouris and Fiehler (2017) participants never reached to the digit that was stimulated, but to another digit of the same hand. This may point to two possible directions of perceptual modulation. First, the enhanced tactile perception at the target hand may be location-unspecific in a way that the perception across the whole hand is uniformly enhanced. Alternatively, the enhanced perception may be location-specific. That is, tactile signals arising at the target digit being enhanced more strongly than signals arising elsewhere on the target hand. In the context of tactile suppression, it has been demonstrated that tactile suppression at the moving limb decrease with increasing distance from the moving body part (Williams, Shenasa, \& Chapman, 1998; Colino \& Binsted, 2016). So far, it is unclear whether and how tactile enhancement is modulated across the target hand depending on the location of the reach target.

In this study, we investigated how location-specific tactile enhancement is at movement-relevant body sites. We asked participants to discriminate a vibrotactile reference stimulus presented on the sternum from a comparison stimulus of variable intensities presented on the unseen left thumb or little finger. The two stimuli were simultaneously presented while participants kept both hands still (baseline condition) or while they performed right-hand reaches to their left thumb or little finger (reaching condition). Thus, in the reaching condition the presentation of the comparison stimulus either coincided with the target or the non-target digit. Based on previous findings (Voudouris \& Fiehler, 2017), we expect that tactile sensitivity during reaching at the target will be enhanced at that hand. If tactile enhancement is location-specific, we expect stronger tactile enhancement at the target than the non-target digit during reaching compared with baseline.

\section{Experiment 1}

\section{Methods}

\section{Participants}

Sixteen healthy and naïve volunteers participated in the study; two of them were excluded from further analyses due to outlier correction (see "Data analysis"). The remaining 14 participants ( 7 males; median age: 22 years, 19-44 years old) were right-handed according to the German translation of the Edinburgh Handedness Inventory (Oldfield, 1971; mean \pm SD: $85 \pm 12$ ). Before the experiment, participants gave their written, informed consent approved by the local ethics committee of the Giessen University. They received 8 euro/hour or course credits for their participation.

\section{Apparatus}

Participants sat in front of a table having their head resting on a chin-rest and their right wrist on a start button. To eliminate any visual information, the experiment was run in a dark room and a black cardboard, attached to the chinrest, occluded the workspace. Suprathreshold vibrotactile stimuli varying in intensity $(250 \mathrm{~Hz}, 50 \mathrm{~ms})$ were generated through custom-made vibrotactile devices (Engineer Acoustics Inc., Florida, USA). An infrared marker attached to the participant's right index fingernail was recorded at $100 \mathrm{~Hz}$ with an Optotrak Certus (NDI, Waterloo, Ontario, Canada). Two foot-pedals were placed on the floor in front of the participants, one for each of their feet. Through these pedals participants gave their responses in the discrimination task. A schematic top-view of the setup is drawn in Fig. 1.

\section{Procedure}

Participants held their left hand still on a table at approximately $45 \mathrm{~cm}$ in front of their chest and $10 \mathrm{~cm}$ to the left of their body midline, with the digits wide apart but in a comfortable configuration. Small pads on the table indicated the location where to place the hand. Because of interindividual differences (different arm, hand, and digits sizes), participants were allowed to adopt the most comfortable position within the indicated region. Their right wrist was resting on the start button approximately $10 \mathrm{~cm}$ in front of the participants and $20 \mathrm{~cm}$ to their right. In each trial, two vibrotactile stimuli were simultaneously presented: a reference stimulus on the sternum, and a comparison stimulus on the dorsal part of either the left thumb or little finger. Participants had to discriminate the intensity of the two stimuli and report whether the comparison stimulus was stronger or weaker than the reference by pressing a foot-pedal. To prevent participants from memorizing the reference stimulus, we presented two reference stimuli 
a

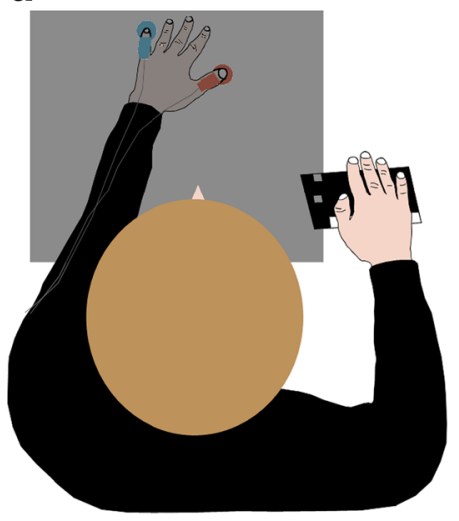

b

\section{stimulation with movement onset \\ during movement}

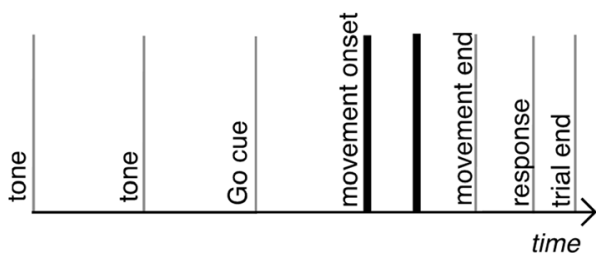

Fig. 1 (a) Top-view of the setup and timeline. The black cardboard is illustrated with the transparent gray box. Rectangular and circular colorful shapes around the participants' digits represent the vibrotactile devices and the target locations, respectively. The foot-pedals are not shown. (b) A trial in the reaching condition started with the presentation of three auditory tones indicating the target-digit, with the last tone being

that differed in intensity: a weak (peak-to-peak displacement of $0.28 \mathrm{~mm}$ ) and a strong (peak-to-peak displacement of 0.34 $\mathrm{mm})$ stimulus. Each of the reference stimuli was combined with one of 11 comparison stimuli of different intensities (peak-to-peak displacement of $0.03,0.09,0.16,0.22,0.28$, $0.34,0.41,0.47,0.53,0.60$, or $0.66 \mathrm{~mm}$ ).

Participants had to perform the tactile discrimination task during a baseline and a reaching condition, and each condition was presented in different blocks of trials. In the baseline condition, each trial started with the press of the start button and the subsequent presentation of three consecutive tones $(800 \mathrm{~Hz}, 50 \mathrm{~ms})$. Two vibrotactile stimuli were simultaneously presented $150 \mathrm{~ms}$ after the last tone, and participants had to indicate whether the comparison stimulus was stronger or weaker than the reference stimulus by pressing the appropriate foot-pedal. In the reaching condition, each trial started with the announcement of the target location (thumb or little) and the subsequent presentation of the 3 tones. Participants had to initiate a right hand reaching movement towards the fingernail of the announced target digit with the onset of the last tone ( $G o$ cue). They were instructed to reach to and touch the target fingernail with their right index fingertip as accurately as possible. No further instructions were given. The vibrotactile stimuli were presented $150 \mathrm{~ms}$ after movement onset as determined by the release of the start button. To impose uncertainty about the timing of stimulation, we also introduced $20 \%$ catch trials, in which the tactile stimulus was presented with movement onset. The psychophysical responses and statistics of these catch trials were not analyzed. After the reaching movement, participants had to indicate whether the comparison stimulus felt stronger or weaker than the reference stimulus by pressing the appropriate foot-pedal. They were instructed to do so as carefully and accurately as possible. also the Go cue. The tactile stimulation occurred either with movement onset ( $20 \%$ of trials) or $150 \mathrm{~ms}$ after movement onset ( $80 \%$ of trials), as indicated by the thick black lines. After the reach, participants responded as to whether the comparison stimulus was stronger or weaker than the reference

The 11 comparison stimuli with the 2 reference stimuli and the 2 stimulation locations resulted in 44 combinations in the baseline condition, whereas in the reaching condition these combinations were extended by the 2 target locations, resulting in 88 combinations. Each of the 44 combinations in the baseline condition was presented 36 times resulting in a total of 1,584 trials. Each of the 88 combinations in the reaching condition was presented 20 times with stimuli after movement onset and 5 times with stimuli at movement onset, resulting in a total of 2,112 trials. Each of the baseline and reaching conditions were presented in 4 separate blocks of trials (thus a total of 8 blocks of trials), with a baseline block either preceding or following a reaching block. Participants performed the experiment in 2 sessions over 2 consecutive days. Each combination of stimuli was presented in a random order within each block, and the presentation of the blocks was counterbalanced across participants. The experiment lasted in total approximately 3 hours for each participant.

\section{Data analysis}

The responses of the comparison stimuli judged as stronger than the reference were fit for each participant to a logistic function using the maximum-likelihood estimation with the function psignift in Matlab (Wichmann \& Hill, 2001). To evaluate the strength of tactile modulation, we estimated the point-of-subjective-equality (PSE) as the $50 \%$ point of the psychometric function. Moreover, we explored the precision of discrimination by examining the just-noticeable-difference (JND), defined as the difference in stimulus' intensity between the $50 \%$ and the $84 \%$ points of the psychometric function. For the baseline condition, we calculated a total of 4 PSE and JND values; more specifically, we calculated one PSE and one JND 
value for each of the 4 combinations of the 2 reference stimuli (weak, strong) and the 2 stimulation locations (thumb, little finger). For the reaching condition, we calculated a total of 8 PSE and JND values; more specifically, we calculated one PSE and one JND value for each of the 8 combinations of the 2 reference stimuli (weak, strong), the 2 stimulation locations (thumb, little finger), and the 2 target locations (thumb, little finger). To examine effects of reaching on the accuracy and precision of discrimination we subtracted each participant's baseline PSE and JND in each of the 4 baseline conditions ( 2 reference stimuli $x 2$ stimulation locations) from his or her respective reaching PSE and JND separately for reaches to the thumb and the little finger. The calculated differences ( $\left.\mathrm{PSE}_{\mathrm{diff}}, \mathrm{JND}_{\mathrm{diff}}\right)$ were averaged across blocks for each participant, and then across participants. These values represent the strength of tactile modulation with negative values indicating tactile enhancement. We expected that tactile signals will generally be enhanced when reaching than in baseline (i.e., negative $\left.\mathrm{PSE}_{\text {diff }}\right)$. Moreover, if tactile enhancement is locationspecific, this enhancement should be stronger when the stimulus and the target location coincide compared to conditions in which they differ. We do not have specific predictions about

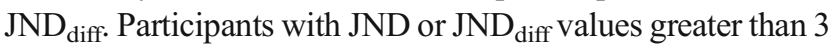
standard deviations of the sample's respective mean were excluded from all analyses $(n=2)$.

We first examined whether the obtained baseline PSEs and JNDs were stable between the 4 blocks and the 2 sessions of trials by using a one-way ANOVA. We then examined whether participants were accurate in the tactile discrimination in the baseline condition. To do so, we tested whether baseline PSEs with the weak and the strong reference stimuli were different from the veridical intensities of the reference stimuli ( 0.28 and $0.34 \mathrm{~mm}$, respectively). Effects of the stimulation location (thumb, little finger), target location (thumb, little finger), and reference intensity (weak, strong) on the $\mathrm{PSE}_{\text {diff }}$ and $\mathrm{JND}_{\text {diff }}$ were examined with a 2 × 2 × 2 repeated measures ANOVA. When sphericity was violated, Greenhouse-Geisser correction was applied. The $p$ value was set to 0.05 . The four experimental conditions and the changes predicted by spatial specificity of tactile stimulations at the target hand are schematically illustrated in Fig. 2.

We also analyzed whether the strength of the change in tactile sensitivity was related to kinematic measures, such as movement speed or endpoint accuracy and precision. However, because we did not find any such relationship, we do not report kinematic results.

\section{Results}

\section{Stability of baseline performance}

Because our baseline data were obtained in different blocks and sessions, we first wanted to confirm that the participants' performance did not differ between blocks and across sessions. The baseline PSEs indeed did not differ between the 4 blocks $\left(F_{3,39}=2.7, p=0.54, \eta^{2}=0.17\right)$ : the average PSE variability (average standard deviation between the 4 blocks \pm standard error of the participants' mean) was $0.07 \pm 0.01 \mathrm{~mm}$ and $0.06 \pm 0.01 \mathrm{~mm}$ for both digits and for the weak and strong reference, respectively. The baseline PSEs were similar across the 2 sessions $\left(F_{1,13}=2.7, p=0.12, \eta^{2}=0.17\right)$. Their variability for the weak reference was $0.07 \pm 0.01 \mathrm{~mm}$ and $0.05 \pm 0.01 \mathrm{~mm}$ for the thumb and little finger, respectively, whereas for the strong reference it was $0.05 \pm 0.01 \mathrm{~mm}$ for both digits. JNDs differed between the 4 baseline blocks $\left(F_{3}\right.$, $\left.{ }_{39}=3.5, p=0.02, \eta^{2}=0.21\right)$, as those in the fourth block were $27 \%$ greater than those in the $3^{\text {rd }}$ block $\left(t_{13}=3.4, p=0.005\right)$. The variability of the JNDs between the 4 baseline blocks was $0.04 \pm 0.01 \mathrm{~mm}$ for both digits and references. The JNDs were similar across the 2 sessions $\left(F_{1,13}=0.6, p=0.43, \eta^{2}=0.04\right)$. Their variability was $0.03 \pm 0.01 \mathrm{~mm}$ and $0.02 \pm 0.02 \mathrm{~mm}$, for both digits and for the weak and strong reference, respectively. These results show that performing the experiment in different blocks and sessions had a negligible impact on the performance of our participants.

\section{Baseline performance}

After establishing that participants had a stable performance between blocks and across sessions, we averaged their performance over the 4 blocks to examine how accurately and precisely they discriminated the two tactile stimuli. The accuracy of the discrimination in the baseline blocks, as reflected by the PSEs, was high when participants were presented with the weak reference stimulus (peak-to-peak displacement of 0.28 $\mathrm{mm}$ ). Indeed, the average baseline PSE was $0.26 \pm 0.01 \mathrm{~mm}$ for the thumb and $0.32 \pm 0.02 \mathrm{~mm}$ for the little finger, and none of them was significantly different from the veridical intensity of the weak reference stimulus (thumb: $p=0.088$; little finger: $p=0.068$ ). When presented with the strong reference (peak-topeak displacement of $0.34 \mathrm{~mm}$ ), participants were accurate for comparison stimuli on their thumb (average PSE of $0.33 \pm$ $0.01 \mathrm{~mm} ; p=0.4$ ) but not for comparison stimuli on their little finger (average PSE of $0.42 \pm 0.02 \mathrm{~mm}, 23 \%$ underestimation; $\left.t_{13}=3.1, p=0.008, \omega^{2}=0.43\right)$. In general, participants underestimated the intensity of stimuli on their little finger compared with the reference stimuli on the sternum $\left(F_{1,13}=\right.$ 8.2, $\left.p=0.013, \eta^{2}=0.38\right)$. Participants' precision of discrimination in baseline, as reflected by the JNDs, was $0.13 \pm$ $0.01 \mathrm{~mm}$ and $0.14 \pm 0.01 \mathrm{~mm}$ for both digits and for the weak and strong reference stimulus, respectively. JNDs were greater when stimuli were compared with the strong reference $\left(F_{1,13}=\right.$ $\left.7.7, p=0.016, \eta^{2}=0.37\right)$ : this was because participants' precision of discrimination was poorer when stimuli on their little finger were compared with the strong than with the weak reference stimulus $\left(t_{13}=2.74, p=0.017, \omega^{2}=0.31\right)$. 


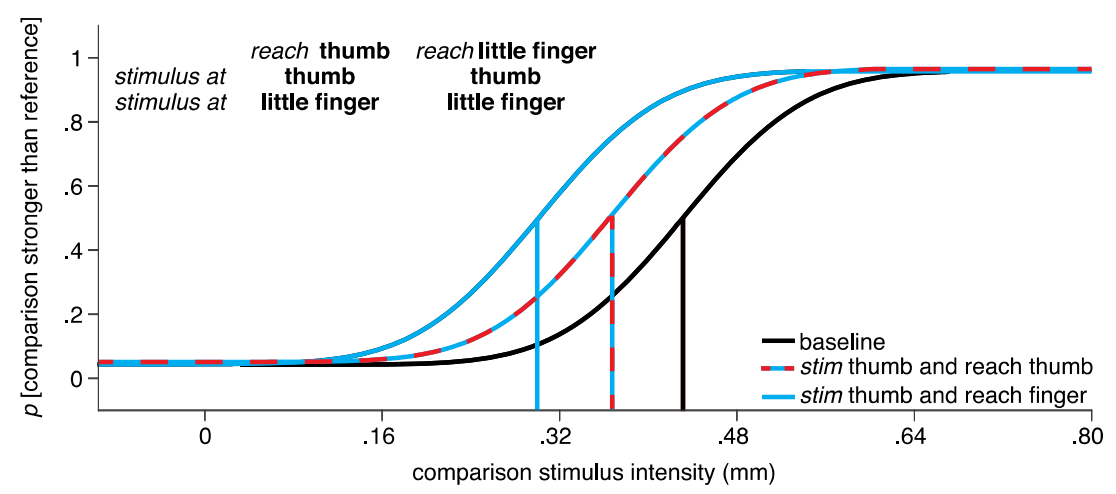

Fig. 2 The predicted psychometric curves for the conditions with stimuli on the thumb. We expect that stimuli on the target hand will be perceived stronger when reaching than during baseline. This will be reflected in a leftward shift of the psychometric functions and thus in lower PSEs (colored compared to black lines). If tactile enhancement is specific to the target location, stimuli on the thumb (shown here) are expected to be perceived even stronger (solid cyan line), thus PSEs will be even lower compared with when reaching to the little finger (cyan-red lines). The same logic applies also for stimuli on the little finger

interaction between stimulus and target location $\left(F_{1,13}=\right.$ $0.01, p=0.9, \eta^{2}=0.001$ ).

After establishing that stimuli on the target hand were enhanced, we further examined our main question, i.e., whether and how location-specific tactile enhancement is at the target hand. We compared the $\mathrm{PSE}_{\text {diff }}$ between reaches to the thumb and to the little finger for each stimulus location. Our results show that there is no modulation of tactile enhancement based on the location of the stimulus and the reach target (all $t<1.2$, $\left.p>0.2, \omega^{2}>0.03\right)$. More specifically, the average difference between reaches to the thumb and the little finger was $-0.01 \pm$ $0.01 \mathrm{~mm}$ for stimuli on the thumb and $-0.02 \pm 0.01 \mathrm{~mm}$ for stimuli on the little finger. We also calculated Bayes factors to examine the probabilities of our hypothesis being correct. More specifically, we compared the hypothesis that tactile enhancement will be stronger for stimuli at the target than the non-target finger. The computed Bayes factors provided no evidence for a difference between target and non-target finger but rather revealed inconclusive results (thumb: $\mathrm{BF}=$ 0.52 ; little finger: $\mathrm{BF}=0.62$ ) based on the guidelines proposed by Jeffreys (1961).

Tactile discrimination was more precise for stimuli during reaching than baseline. Indeed, $J \mathrm{ND}_{\text {diff }}$ were negative in all conditions except for when stimuli were presented on the thumb and reaches were done to the little finger (Fig. 4b). More specifically, $\mathrm{JND}_{\text {diff }}$ was $-0.03 \pm 0.01 \mathrm{~mm}$ for stimuli and reaches to the thumb $\left(t_{13}=-2.3, p=0.037, \omega^{2}=0.23\right)$, $-0.01 \pm 0.01 \mathrm{~mm}$ for stimuli on the thumb and reaches to the little finger $\left(t_{13}=-1.3, p=0.202, \omega^{2}=0.04\right),-0.04 \pm$ $0.01 \mathrm{~mm}$ for stimuli and reaches to the little finger $\left(t_{13}=\right.$ $-5.6, p<0.001, \omega^{2}=0.57$ ), and $-0.04 \pm 0.01 \mathrm{~mm}$ for stimuli on the little finger and reaches to the thumb $\left(t_{13}=-4.5, p=\right.$ $\left.0.001, \omega^{2}=0.68\right)$. Similar to the results of PSE $\mathrm{diff}_{\text {, we found a }}$ general effect of reaching in $\mathrm{JND}_{\text {diff }}$ but no differences in $\mathrm{JND}_{\text {diff }}$ between the target digit and the non-target digit (all $t$ $<1.4, p>0.1, \omega<0.06)$. 


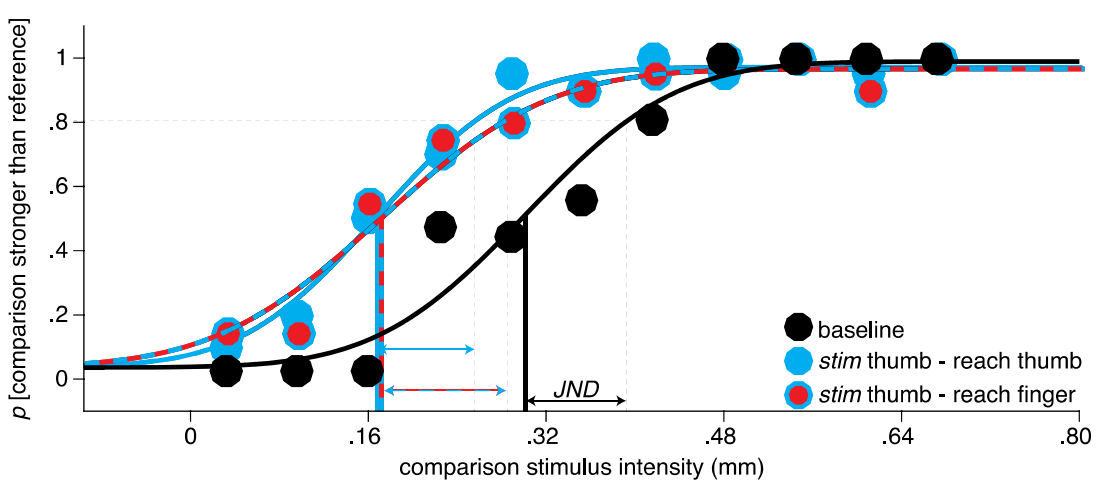

Fig. 3 Psychometric curves of one participant with weak reference stimuli for the baseline (black), for comparison stimuli on the thumb and reaches to the thumb (cyan outline with cyan filling), and for comparison stimuli on the thumb and reaches to the little finger (cyan outline with red filling). The PSEs (vertical lines) are lower in the

\section{Discussion}

In this experiment we investigated whether tactile enhancement is location-specific, i.e., if it is stronger at the target than the non-target location of the same hand. Participants discriminated a reference vibrotactile stimulus on their sternum from a comparison stimulus on their left thumb or little finger. The stimuli were presented simultaneously either while both hands were still (baseline) or while reaching with their right hand towards their left thumb or little finger. Thus, the comparison stimulus was presented on either the target or the non-target digit of the target hand. Our results showed that tactile stimuli were enhanced on the target hand during reaching compared to baseline, confirming our previous findings (Voudouris \& Fiehler, 2017). However, the strength of tactile enhancement did not vary with the target location, i.e., tactile enhancement was similarly strong when reaching to the target and the non-target digit. This suggests that tactile enhancement may have low movement-related spatial specificity (at least across the target hand).

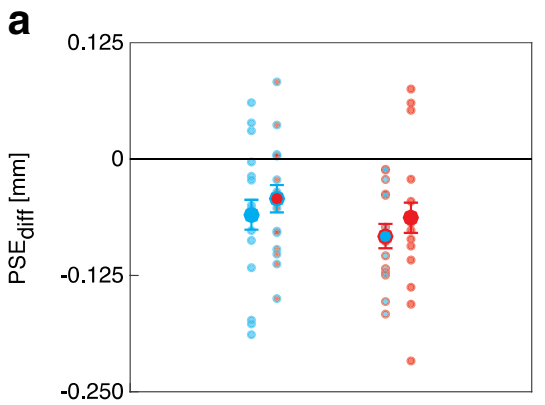

Fig. 4 The difference in tactile sensitivity between baseline and reaching as reflected by changes in (a) PSE, and (b) JND. Negative values indicate lower values when reaching as compared to baseline, suggesting tactile enhancement. Cyan and red outlines represent stimuli on the thumb and little finger, respectively. Cyan and red fillings represent reaches to the reaching conditions than in the baseline, but there seems to be no modulation depending on where the participant reaches. The horizontal arrows show the JND, as the difference in stimulus intensity between the $50 \%$ and $84 \%$ of the psychometric function

\section{Experiment 2}

One could argue that the lack of spatial specificity may arise because participants always expected and received a stimulus on their target hand. Therefore, this expectation of stimulation at both digits may have led to a spatially unspecific tactile enhancement. At the same time, stimulation on the target hand in every trial may have caused a systematic enhancement that cascaded from one trial to the next. To address these issues, we conducted a second experiment. Participants received tactile stimuli not only on their left thumb or little finger but also on their left upper arm. Reaches were again performed only to their left thumb or little finger. Importantly, half of the trials included stimulations at the target hand and the other half included stimulations at the left upper arm, thus providing equal stimulus probabilities for the upper arm and the hand. In addition, experiment 2 can inform about the spatial specificity of tactile enhancement by comparing tactile sensitivity on the upper arm and the target hand. If stimuli on the upper arm are enhanced during reaching to the hand, it would imply that tactile enhancement generalizes across the target arm. On the other hand, if stimuli on the upper arm are less or not

b

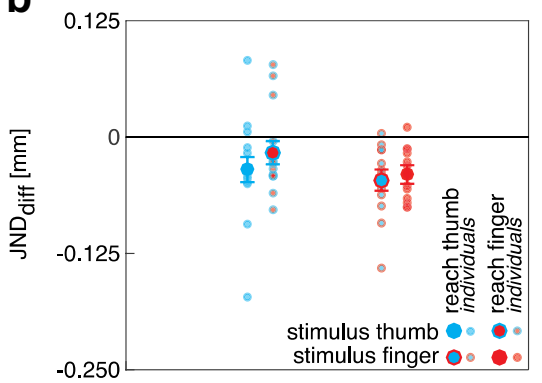

thumb and little finger, respectively. The tactile enhancement effect for the PSE was evident in all conditions, whereas for the JND we observed it in 3 of the 4 conditions. Small dots indicate the values of the individual participants for each condition. Error bars represent the standard error of the participants' mean 
enhanced during reaching, it would suggest a spatial gradient showing decreased tactile enhancement with increased distance from the reach goal.

\section{Methods}

Sixteen right-handed participants (median age: 23.5 years, 18 34 years old) joined the experiment. Two of them were excluded (see "Data analysis" of experiment 1), resulting in a total sample of 14 participants. Except for the details mentioned below, the apparatus, procedure, and data analysis were the same as in experiment 1 . We now presented only the weak reference stimulus (peak-to-peak displacement $0.28 \mathrm{~mm}$ ) together with one of nine comparison stimuli (peak-to-peak displacement of $0.03,0.09,0.16,0.22,0.28,0.34,0.41,0.47$, or $0.53 \mathrm{~mm}$ ). The two vibrotactile stimuli were presented simultaneously $150 \mathrm{~ms}$ after participants started reaching. No stimuli were presented at the time of movement onset. The reference stimulus was presented on the sternum and the comparison stimulus either on the left thumb, or the left little finger, or the left upper arm (lower part of the deltoid muscle). Participants were only instructed about where to reach but not about where the stimulus will be presented. For the reaching block, we ran a total of 432 trials, with half of them including a stimulus on the upper arm (216 trials), and the other half a stimulus on the target hand (108 trials with stimuli on the thumb, and 108 trials with stimuli on the little finger), whereas for the baseline block we ran 216 trials.

\section{Results}

\section{Baseline performance}

The average baseline PSE was $0.16 \pm 0.02 \mathrm{~mm}$ for the thumb, $0.23 \pm 0.02 \mathrm{~mm}$ for the little finger, and $0.27 \pm 0.02 \mathrm{~mm}$ for the upper arm. Participants' baseline PSEs were not similar to the veridical intensity of the reference stimulus $\left(F_{2,26}=8.15, p=\right.$ $\left.0.002, \eta^{2}=0.38\right)$. Indeed, participants significantly overestimated the intensity of the stimuli on their thumb and little finger but not on their arm (thumb: $t_{13}=-6.4, p<0.001$, $\omega^{2}=0.74$; little finger: $t_{13}=-2.6, p=0.02, \omega^{2}=0.29$; arm: $t_{13}$ $\left.=-0.7, p=0.49, \omega^{2}=0.03\right)$. The overestimation of the stimuli on the thumb was stronger when compared to that on the little finger $\left(t_{13}=-3.8, p=0.002, \omega^{2}=0.49\right)$ and to the perception of stimuli on the upper $\operatorname{arm}\left(t_{13}=-3.5, p=0.004, \omega^{2}=0.44\right)$. Participants' precision of discrimination in the baseline, as reflected by the JNDs, was $0.09 \pm 0.01 \mathrm{~mm}$ for stimuli on the thumb, $0.12 \pm 0.01 \mathrm{~mm}$ for stimuli on the little finger, and 0.08 $\pm 0.01 \mathrm{~mm}$ for stimuli on the upper arm. The baseline JNDs were influenced by the stimulus location $\left(F_{2,26}=3.86, p=\right.$ $\left.0.034, \eta^{2}=0.22\right)$ : the baseline JND for stimuli on the upper arm was significantly smaller only compared with that for stimuli on the little finger $\left(t_{13}=2.3, p=0.034, \omega^{2}=0.23\right)$.
Modulation of tactile perception during reaching

Tactile perception was modulated depending on the stimulus location $\left(F_{2,26}=12.69, p<0.001, \eta^{2}=0.49\right)$ : it was different for stimuli between thumb and little finger $\left(t_{13}=3.1, p=\right.$ $\left.0.008, \omega^{2}=0.38\right)$, between thumb and upper arm $\left(t_{13}=\right.$ 2.6, $p=0.02, \omega^{2}=0.29$ ), and between little finger and upper $\operatorname{arm}\left(t_{13}=-4.2, p=0.001, \omega^{2}=0.54\right)$. Tactile enhancement, as reflected by the $\mathrm{PSE}_{\text {diff }}$ values being lower than zero, was again evident for stimuli on the little finger $(-0.09 \pm 0.02 \mathrm{~mm}$; $\left.t_{13}=-3.5, p=0.004, \omega^{2}=0.44\right)$. However, the effect did not reach significance levels for stimuli on the thumb $(-0.03 \pm$ $0.02 \mathrm{~mm} ; t_{13}=-1.7, p=0.099, \omega^{2}=0.12$ ). Stimuli on the upper arm also were not enhanced $\left(0.01 \pm 0.01 \mathrm{~mm} ; t_{13}=1.7\right.$, $p=0.098, \omega^{2}=0.12$; Fig. 5a). More specifically, $\operatorname{PSE}_{\text {diff }}$ was $-0.02 \pm 0.02 \mathrm{~mm}$ for stimuli and reaches to the thumb $\left(t_{13}=\right.$ $\left.-1.1, p=0.25, \omega^{2}=0.01\right),-0.05 \pm 0.02 \mathrm{~mm}$ for stimuli on the thumb and reaches to the little finger $\left(t_{13}-2.1, p=0.054, \omega^{2}=\right.$ $0.19),-0.10 \pm 0.03 \mathrm{~mm}$ for stimuli and reaches to the little finger $\left(t_{13}=-3.2, p=0.007, \omega^{2}=0.39\right),-0.08 \pm 0.02 \mathrm{~mm}$ for stimuli on the little finger and reaches to the thumb $\left(t_{13}=-3.6\right.$, $\left.p=0.003, \omega^{2}=0.46\right), 0.03 \pm 0.01 \mathrm{~mm}$ for stimuli on the upper arm and reaches to the thumb $\left(t_{13}=2.2, p=0.039, \omega^{2}=0.21\right)$, and $0.00 \pm 0.01 \mathrm{~mm}$ for stimuli on the upper arm and reaches to the little finger $\left(t_{13}=0.2, p=0.815, \omega^{2}=0.07\right)$.

Importantly, tactile enhancement on the little finger was similar for reaches to the little finger and for reaches to the thumb $\left(t_{13}=1.2, p=0.249, \omega^{2}=0.03\right)$, providing additional evidence that tactile enhancement is not spatially specific on the target hand. The calculated Bayes-factor for stimuli on the little finger was 0.61 and for stimuli on the thumb it was 0.95 .

The precision of tactile discrimination, as reflected by the $\mathrm{JND}_{\text {diff, }}$, was influenced by the stimulus location $\left(F_{2,26}=4.3\right.$, $\left.p=0.024, \eta^{2}=0.249\right)$ : it was better for stimuli on the little finger than the thumb $\left(t_{13}=2.7, p=0.018, \omega^{2}=0.31\right.$; Fig. $5 \mathrm{~b})$. In general, the precision of discrimination was improved during reaching for stimuli on the little finger $\left(t_{13}=-4.6, p<\right.$ $\left.0.001, \omega^{2}=0.59\right)$, but not for stimuli on the thumb $\left(t_{13}=0.1, p\right.$ $\left.=0.884, \omega^{2}=0.07\right)$ and on the upper $\operatorname{arm}\left(t_{13}=-1.9, p=\right.$ $\left.0.068, \omega^{2}=0.15\right)$. More specifically, $\mathrm{JND}_{\mathrm{diff}}$ was $-0.005 \pm$ $0.01 \mathrm{~mm}$ for stimuli and reaches to the thumb $\left(t_{13}=-0.4, p=\right.$ $\left.0.583, \omega^{2}=0.06\right), 0.008 \pm 0.01 \mathrm{~mm}$ for stimuli on the thumb and reaches to the little finger $\left(t_{13}=0.6, p=0.501, \omega^{2}=0.06\right)$, $-0.03 \pm 0.007 \mathrm{~mm}$ for stimuli and reaches to the little finger $\left(t_{13}=-4.3, p=0.001, \omega^{2}=0.55\right),-0.03 \pm 0.01 \mathrm{~mm}$ for stimuli on the little finger and reaches to the thumb $\left(t_{13}=-3.4, p=\right.$ $\left.0.004, \omega^{2}=0.43\right),-0.02 \pm 0.01 \mathrm{~mm}$ for stimuli on the upper arm and reaches to the thumb $\left(t_{13}=-1.9, p=0.08, \omega^{2}=0.15\right)$, and $-0.01 \pm 0.01 \mathrm{~mm}$ for stimuli on the upper arm and reaches to the little finger $\left(t_{13}=-1.9, p=0.071, \omega^{2}=0.15\right)$. We found no differences in $\mathrm{JND}_{\text {diff }}$ between the target digit and the nontarget digit (all $t<0.9, p>0.3, \omega<0.01$ ). 


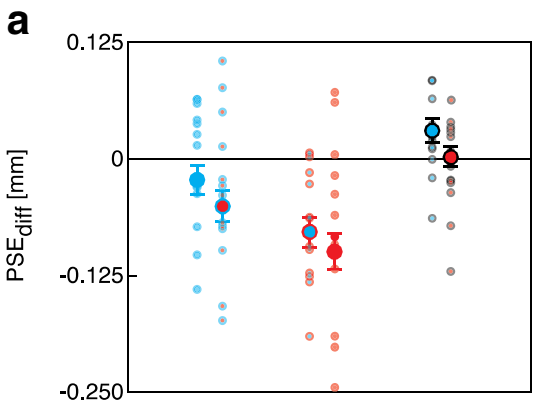

Fig. 5 The difference in tactile sensitivity between baseline and reaching as reflected by changes in (a) PSE, and (b) JND. Cyan, red and black outlines represent stimuli on the thumb, little finger and upper arm,

\section{Discussion}

In experiment 2, we examined whether the low spatial specificity of tactile enhancement found in experiment 1 was due to the stimulation always occurring at the target hand. In experiment 2, half of the trials included a stimulus at the left target hand and the other half of the trials included a stimulus on the left upper arm; thus, participants could not predict on which of the two locations the stimulus would be presented. Similar to experiment 1 , tactile modulation in experiment 2 was stronger on the little finger compared to the thumb. In contrast to experiment 1 though, tactile enhancement was now evident for stimuli on the little finger but did not reach significance for stimuli on the thumb. The weaker tactile modulation on the thumb may be due to differences in the transmission of cutaneous vibrations that are weaker on the thumb compared to the other digits (Shao, Hayward, \& Visell, 2016). In addition, the fact that stimuli on the thumb were not enhanced in experiment 2 is partly caused by the low thresholds for the thumb in the baseline condition, leaving hardly room for tactile enhancement. Indeed, in experiment 2 the baseline PSE on the thumb was $40 \%$ lower compared with that obtained for the weak reference in experiment 1 ( 0.16 vs. $0.26 \mathrm{~mm})$, and importantly this PSE value $(0.16 \mathrm{~mm})$ corresponds to the third weakest stimulus of the range of stimuli we presented. It is notable that not only the thumb baseline PSE was lower in experiment 2 but also the one of the little finger ( 0.32 and $0.23 \mathrm{~mm}$ in experiment 1 and 2, respectively). Such discrepancy in the baseline PSEs might have been caused by the arm and digit posture being slightly different between the two experiments (see "Procedure"), which has been shown to influence tactile sensitivity on the digits (Heed, Backhaus, \& Roder, 2012). Additional differences between the two experiments also might have played a role, for instance that we used only one reference stimulus instead of two and presented less repetitions per condition in experiment 2 .

Importantly, we show again that tactile enhancement has low spatial resolution within the target hand. Indeed, the clear and systematic enhancement of stimuli on the little finger was not stronger when reaching to the little finger compared with

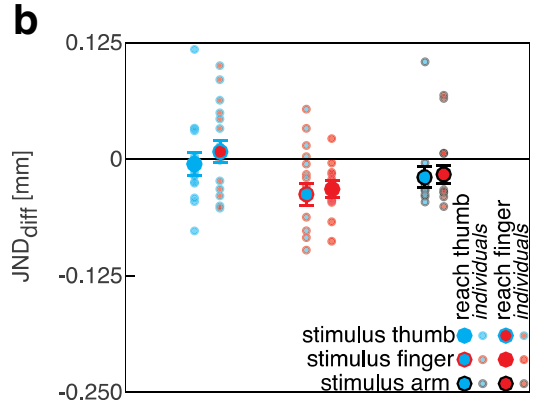

respectively. Cyan and red fillings represent reaches to the thumb and little finger, respectively. Details same as in Fig. 4

when reaching to the thumb. Given that participants did not always expect a stimulus on their target hand, our results confirm the findings of experiment 1 and are in line with the notion that tactile enhancement has low spatial resolution within the target hand.

In our second experiment, we also were able to further examine the gradient of tactile enhancement within the target limb. We found that tactile stimuli on the upper arm are not enhanced when reaching to the hand. Instead, we found a systematic suppression of the stimuli presented on the upper arm, but only when reaching to the thumb. Although we do not have a clear explanation for this effect, it makes clear that participants do not enhance their tactile sensitivity across their whole target arm when reaching to their hand. Hence, tactile sensitivity increases at the target hand but does not generalize to the whole arm, providing first evidence for the general boundaries of tactile enhancement.

\section{General discussion}

In this study, we examined whether tactile enhancement is location-specific, i.e., if it is stronger at the target than the non-target location of the same hand. We did find that stimuli on the target hand were in general perceived stronger when reaching than during baseline, supporting our previous results (Voudouris \& Fiehler, 2017). The strength of tactile enhancement did not vary with the target location, i.e., tactile enhancement was similarly strong when reaching to the target or the non-target digit of the same hand. In contrast to the target hand, there was no evidence for tactile enhancement at the upper arm. This suggests that tactile enhancement has low movement-related spatial specificity across the target hand but shows a spatial gradient with farther distance.

To examine the specificity of tactile enhancement, we first confirmed our previous findings that tactile sensitivity is enhanced at the unseen target hand during reaching (Voudouris \& Fiehler, 2017). Accordingly, we observed overall lower PSEs during reaching than baseline. This suggests that stimuli on the target hand were perceived stronger when reaching to 
that hand than when discriminating the stimuli at rest. Such an effect is unlikely to occur solely because of the reaching movement itself, because we have shown previously that tactile enhancement on the target hand is evident only when reaching to that hand and absent when reaching to an external visual target (Voudouris \& Fiehler, 2017). We argue that tactile signals are enhanced at movement-relevant locations, such as reach goals.

One might expect that controlling a movement while processing two simultaneously presented tactile signals would lead to deteriorated tactile discrimination performance because of additional processing demands. Our results show that the precision of tactile discrimination was not impaired during reaching. Indeed, in both experiments the precision of tactile discrimination was better when reaching than in baseline, as reflected by the generally lower JNDs.

Our findings about tactile enhancement are in line with previous evidence suggesting a tight link between movement planning or execution and tactile enhancement (Forster \& Eimer, 2007; Juravle et al., 2016). Such a link has been proposed by the premotor theory of attention, according to which humans improve their sensory processing at locations towards where they plan a movement (Rizzolatti, Riggio, Dascola, \& Umilta, 1987; but see also Smith \& Schenk, 2012). For instance, visual discrimination is more accurate (Moehler \& Fiehler, 2014; Deubel, Schneider, \& Paprotta, 1998) and tactile detection is faster (Rorden et al., 2002) at locations to which an eye or hand movement is being planned. In contrast, an ERP study, in which participants performed reaching movements to their own hand similar to those in our study, revealed enhanced somatosensory N140 amplitudes to tactile stimuli on the moving hand but not to stimuli on the target hand (Forster \& Eimer, 2007). The latter findings suggest that for reaching movements towards somatosensory targets humans prioritize the processing of tactile signals presented on their moving arm instead of signals presented on their static target hand (but see also Eimer, Forster, van Velzen, \& Prabhu, 2005). Here, we found tactile enhancement at the somatosensory reach goal, which speaks in favor of enhanced sensory processing at the target hand. This difference between our results and that by Forster and Eimer (2007) might be attributed to the fact that our stimuli were presented during movement execution but not during movement planning, as in their study. Tactile enhancement at the target hand during movement execution may indicate that such sensory up-regulation can occur at the reach goal also after movement preparation.

Our main question was how location-specific tactile enhancement is. So far, the spatial characteristics of tactile enhancement during reaching are widely unknown. If tactile signals are enhanced when they arise at the target location, one might expect stronger enhancement when the stimulus and target location coincide. Our current results show that this is not the case for stimuli on the target hand.
One possible reason for the lack of a location-specific tactile enhancement within the target hand might be that the location where stimuli were presented on the hand as well as the target locations were too close together. For instance, in the context of tactile suppression it has been shown that tactile sensitivity is modulated by the location of the stimulation with respect to the moving body part. Indeed, the strength of suppression decreased with the distance between the stimulus location and the moving body part showing only negligible differences in sensitivity for small distances within the same hand (digit vs. palm; Williams et al., 1998). In our experiment 2, we provide evidence that tactile enhancement has some spatial specificity, as we did not find any tactile enhancement for stimuli on the left upper arm while reaching to the left hand. Because we did not test for tactile sensitivity for locations at the palm or forearm, we cannot further comment about how broad is the spatial gradient of tactile enhancement.

It has been shown that cutaneous vibrations on the digits propagate within the hand (Shao et al., 2016). However, these propagations are not strong enough to cover areas farther than the immediate neighboring digit. In our experiment, the stimulus was applied either to the thumb or little finger, the two digits that are farthest apart. Therefore, it is unlikely that stimuli presented on the thumb would be felt on the little finger, and vice versa.

What is the purpose of tactile enhancement? Enhancing the perception of tactile stimuli at the movement target may facilitate the use of somatosensory information for successfully planning and guiding a movement. This is particularly important because in our experiment visual information was withdrawn. Indeed, there is evidence that tactile sensitivity increases when vision is available compared with when it is not (Colino, Lee, \& Binsted, 2016; Cardini, Longo, Driver, \& Haggard, 2012). Yet, we intentionally removed visual information so that participants had to rely only on somatosensory signals from their target hand to derive the target location. Moreover, somatosensory signals from the target hand are important because they provide sensory feedback at the time of contact about whether the reaching movement was correctly performed. Therefore, enhancing tactile perception at a movementrelevant location can be functionally important for a successful movement, at least when visual information is not available. In line with the importance of tactile enhancement, we have recently shown that tactile suppression is stronger when reaching to body than external targets, which suggests that humans may suppress irrelevant tactile stimuli on the moving limb to release resources for processing task-relevant (somatosensory) signals (Gertz, Voudouris, \& Fiehler, 2017).

Lastly, when participants performed the tactile discrimination task in the baseline condition, they were not always accurate when perceiving the tactile stimuli. Indeed, in 
experiment 1 and for the strong reference, participants underestimated the intensity of the stimuli on their little finger, whereas in experiment 2 they overestimated the intensity of the stimuli on their thumb. The possibility that participants did not correctly localize which of the two digits was stimulated is unlikely, as no participant reported such difficulties. Yet, differences in tactile sensitivity of different digits may partly explain these effects. For instance, tactile acuity becomes gradually lower from the index to the middle and from the middle to the ring finger (Vega-Bermudez \& Johnson, 2001). Moreover, tactile processing on the digits appears to depend on many factors, such as arm and finger posture (Haggard, Kitadono, Press, \& Taylor-Clarke, 2006; Heed et al., 2012; Heed \& Azanon, 2014). Nevertheless, this effect does not jeopardize our conclusions, as our measures of tactile modulation are all relative to the tactile sensitivity obtained in the baseline. In experiment 1 , discrimination thresholds on the little finger tended to be slightly higher than those on their thumb. This is at odds with previous findings showing that two-point discrimination thresholds on the little finger are lower than those on the thumb (Kalisch, Tegenthoff, \& Dinse, 2007).

In sum, we demonstrate that tactile stimuli on the target hand are generally enhanced during reaching when this hand serves as movement target. Tactile enhancement has some spatial specificity, as stimuli on the upper target arm were not enhanced. Yet, tactile enhancement seems to spread throughout the target hand and has low movement-related spatial specificity.

Acknowledgements The authors thank Hanna Gertz for assistance in data collection. This work was supported by the German Research Foundation (DFG) TRR 135. Participants' responses to the stimuli are available online at https://zenodo.org/record/822257.

\section{References}

Buckingham, G., Carey, D. P., Colino, F. L., deGrosbois, J., \& Binsted, G. (2010). Gating of vibrotactile detection during visually guided bimanual reaches. Experimental Brain Research, 201, 411-419.

Cardini, F., Long, M. R., Driver, J., \& Haggard, P. (2012). Rapid enhancement of touch from non-informative vision of the hand. Neuropsychologia, 50, 1954-1960.

Chapman, C. E., Bushnell, M. C., Miron, D., Duncan, G. H., \& Lund, J. P. (1987). Sensory perception during movement in man. Experimental Brain Research, 68, 516-524.

Colino, F. L., \& Binsted, G. (2016). Time course of tactile gating in a reach-to-grasp and lift task. Journal of Motor Behavior, 48, 390400 .

Colino, F. L., Lee, J. H., \& Binsted, G. (2016). Availability of vision and tactile gating: Vision enhances tactile sensitivity. Experimental Brain Research, 235, 341-348.

Deubel, H., Schneider, W. X., \& Paprotta, I. (1998). Selective dorsal and ventral processing: Evidence for a common attentional mechanism in reaching and perception. Visual Cognition, 5, 81-107.
Eimer, M., Forster, B., van Velzen, J., \& Prabhu, G. (2005). Covert manual response preparation triggers attentional shifts: ERP evidence for the premotor theory of attention. Neuropsychologia, 43, 957-966.

Forster, B., \& Eimer, M. (2007). Covert unimanual response preparation triggers attention shifts to effectors rather than goal locations. Neuroscience Letters, 419, 142-146.

Gertz, H., Voudouris, D., \& Fiehler, K. (2017). Reach-relevant somatosensory signals modulate tactile suppression. Journal of Neurophysiology, 11, 2262-2268.

Haggard, P., Kitadono, K., Press, C., \& Taylor-Clarke, M. (2006). The brain's fingers and hands. Experimental Brain Research, 172, 94 102.

Heed, T., \& Azanon, E. (2014). Using time to investigate space: A review of tactile temporal order judgments as a window onto spatial processing in touch. Frontiers in Psychology, 5, 76.

Heed, T., Backhaus, J., \& Roder, B. (2012). Integration of hand and finger location in external spatial coordinates for tactile localization. Journal of Experimental Psychology: Human Perception and Performance, 38, 386-401.

Jeffreys, H. (1961). Theory of probability. In N. F. Mott, E. C. Bullard, \& D. H. Wilkinson (Eds.), The international series of monographs on physics (3rd ed.). Oxford: Oxford University Press.

Johansson, R. S., Westling, G., Backstrom, A., \& Flanagan, J. (2001). Eye-hand coordination in object manipulation. Journal of Neuroscience, 21, 6917-6932.

Juravle, G., \& Deubel, H. (2009). Action preparation enhances the processing of tactile targets. Experimental Brain Research, 198, 301311.

Juravle, G., Heed, T., Spence, C., \& Roder, B. (2016). Neural correlates of tactile perception during pre-, peri-, and post-movement. Experimental Brain Research, 234, 1293-1305.

Juravle, G., McGlone, F., \& Spence, C. (2013). Context-dependent changes in tactile perception during movement execution. Frontiers in Psychology, 4, 913.

Kalisch, T., Tegenthoff, M., \& Dinse, H. R. (2007). Differential effects of synchronous and asynchronous multifinger coactivation on human tactile performance. BMC Neuroscience, 8, 58 .

Moehler, T., \& Fiehler, K. (2014). Effects of spatial congruency on saccade and visual discrimination performance in a dual-task paradigm. Vision Research, 105, 100-111.

Oldfield, R. C. (1971). The assessment and analysis of handedness: The Edinburgh inventory. Neuropsychologia, 9, 97-113.

Parkinson, A., Plukaard, S., Pears, S. L., Newport, R., Dijkerman, C., \& Jackson, S. R. (2011). Modulation of somatosensory perception by motor intention. Cognitive Neuroscience, 2, 47-56.

Rizzolatti, G., Riggio, L., Dascola, I., \& Umilta, C. (1987). Reorienting attention across the horizontal and vertical meridians: Evidence in favor of a premotor theory of attention. Neuropsychologia, 25, 3140.

Rorden, C., Greene, K., Sasine, G. M., \& Baylis, G. C. (2002). Enhanced tactile performance at the destination of an upcoming saccade. Current Biology, 12, 1249-1434.

Shao, Y., Hayward, V., \& Visell, Y. (2016). Spatial patterns of cutaneous vibration during whole-hand haptic interactions. Proceeding of the National Academy of Sciences of the United States of America, 113, 4188-4193.

Smeets, J. B. J., Hayhoe, M. M., \& Ballard, D. H. (1996). Goal-directed arm movements change eye-head coordination. Experimental Brain Research, 109, 434-440.

Smith, D. T., \& Schenk, T. (2012). The premotor theory of attention: Time to move on? Neuropsychologia, 50, 1104-1114.

van Ede, F., van Doren, T. I., Damhuis, J., de Lange, F. P., \& Maris, E. (2015). Movement preparation improves touch perception without awareness. Cognition, 137, 189-195.

Vega-Bermudez, F., \& Johnson, K. O. (2001). Differences in spatial acuity between digits. Neurology, 56, 1389-1391. 
Voss, M., Ingram, J. N., Wolpert, D. M., \& Haggard, P. (2008). Mere expectation to move causes attenuation of sensory signals. PLoS One, 3, e2866.

Voudouris, D., \& Fiehler, K. (2017). Enhancement and suppression of tactile signals during reaching. Journal of Experimental Psychology: Human Perception and Performance, 43, 1238-1248.

Voudouris, D., Smeets, J. B. J., \& Brenner, E. (2016). Fixation biases towards the index finger in almost natural grasping. PLoS One, 11, e0146864.

Wichmann, F. A., \& Hill, N. J. (2001). The psychometric function: I. Fitting, sampling, and goodness of fit. Perception \& Psychophysics, 63, 1293-1313.
Williams, S. R., \& Chapman, C. E. (2002). Time course and magnitude of movement-related gating of tactile detection in humans. III Effect of motor tasks. Journal of Neurophysiology, 88, 1968-1979.

Williams, S. R., Shenasa, J., \& Chapman, C. E. (1998). Time course and magnitude of movement-related gating of tactile detection in humans. I. Importance of stimulus location. Journal of Neurophysiology, 79, 947-963.

Wolpert, D. M., \& Flanagan, J. R. (2001). Motor prediction. Current Biology, 11, R729-R732. 